\title{
REFRACTIVE INDEX OF DIAMAGNETIC KCI SOLUTION IN ABSENCE AND IN PRESENCE OF NON UNIFORM MAGNETIC FIELD
}

\author{
PRATIK R. PATANKAR
}

Department of Physics, Fergusson College, India

\begin{abstract}
At room temperature refractive index of diamagnetic $\mathrm{KCl}$ solution at one, two, three and four molal concentration was obtained in absence and in presence of non uniform magnetic field. It was observed that Refractive index of diamagnetic KCl solution at one molal concentration was more in presence of non uniform magnetic field than in absence of magnetic field. It has been found that Refractive index of two, three and four molal concentration of $\mathrm{KCl}$ solution was lesser in presence of non uniform magnetic field than in absence of magnetic field. Due to Fermat principle and non uniform density of solution, it was concluded that refractive index of diamagnetic KCl solution in presence of non uniform magnetic field is lesser than in absence of magnetic field. Using hollow glass prism the refractive index of diamagnetic $\mathrm{KCl}$ solution in presence and in absence of magnetic field was found to increase with increase in molal concentration of $\mathrm{KCl}$ solution.
\end{abstract}

KEYWORDS: Refractive Index, Diamagnetism, $\mathrm{KCl} \&$ Non Uniform Magnetic Field

Received: Jun 17, 2021; Accepted: Jul 07, 2021; Published: Jul 16, 2021; Paper Id.: IJPRDEC20211

\section{INTRODUCTION}

Using Spectrometer, Hollow glass prism and Monochromatic sodium source as a light source refractive index of diamagnetic $\mathrm{KCl}$ solution at 1,2,3 and 4 molal concentration was obtained at room temperature. Prism formula and principle of Shuster's method was used to calculate refractive index of the solution [6] [7]. It was found that refractive index of $\mathrm{KCl}$ solution at one molal concentration was more in presence of non uniform magnetic field than in absence of magnetic field. Whereas refractive index of 2, 3 and 4 molal concentration of $\mathrm{KCl}$ solution was lesser in presence of non uniform magnetic field than in absence of magnetic field. In non uniform magnetic field, the electrons orbiting in their orbits are either speed up or slow down so as to increase net magnetic moment by amount $\left(e^{2} R^{2} B\right) / 4 M$ in opposite direction of magnetic field [2]. Due to this, the molecules in diamagnetic solution move from region of stronger magnetic field to weaker magnetic field and create non uniform density in the solution [1]. Thus, some areas in the medium have density rarer than density of medium that would have been in absence of magnetic field. According to Fermat principle the area in the medium where density is lowest is the path of light chosen to travel [3]. There was increase in velocity of light in the medium when placed between the bar magnets. This might be the reason for decrease in refractive index of diamagnetic $\mathrm{KCl}$ solution at 2, 3 and 4 molal concentration [6]. The increase in refractive index of 1 molal $\mathrm{KCl}$ solution in presence of non uniform magnetic field maybe due to contamination of medium or solution might have larger temperature or may be due to instrumental error [7][8]

\section{EXPERIMENT}

At room temperature 1, 2, 3 and 4 molal $\mathrm{KCl}$ solution were prepared separately. Using spirit level horizontal 
alignment of telescope, collimator and prism table was done. Prism filled with one molal $\mathrm{KCl}$ solution was placed on prism table with its base parallel to collimator and telescope. Using Shuster's method angle of minimum deviation was obtained [9]. To avoid error procedure was repeated 5 times. By using mean of angle of minimum deviation and substituting angle of prism $\mathrm{A}=60^{\circ}$ prism formula

$$
\mu=\operatorname{Sin}\left\{\left[A+\int \mathrm{m}\right] / 2\right\} \quad[10]
$$

$$
\operatorname{Sin}[\mathrm{A} / 2]
$$

Reduces to equation $\mu=2 * \operatorname{Sin}[(60+[\mathrm{m}) / 2] \ldots \ldots \ldots$ (1) Where $\mu$ is the refractive index of desired medium. By using Equation (1) refractive index of one molal $\mathrm{KCl}$ solution in absence of magnetic field was obtained. Similar procedure was followed to compute refractive index of 2, 3 and 4 molal $\mathrm{KCl}$ solution in absence of magnetic field. As shown in figure(1) two bar magnets having magnetic field at surface 1500 gauss were used as a source of non uniform magnetic field. With help of gauss meter it was found that magnetic field above the centre of prism table to be 50 gauss. The prism filled with one molal diamagnetic $\mathrm{KCl}$ solution was placed on prism table between the magnets. Refractive index was obtained by using Shuster's method and prism formula [9] [10]. Similarly, refractive index of 2, 3 and 4 molal concentration of KCl solution was calculated in presence of non uniform magnetic field at room temperature.Observation table used to calculate refractive index of medium are given below.

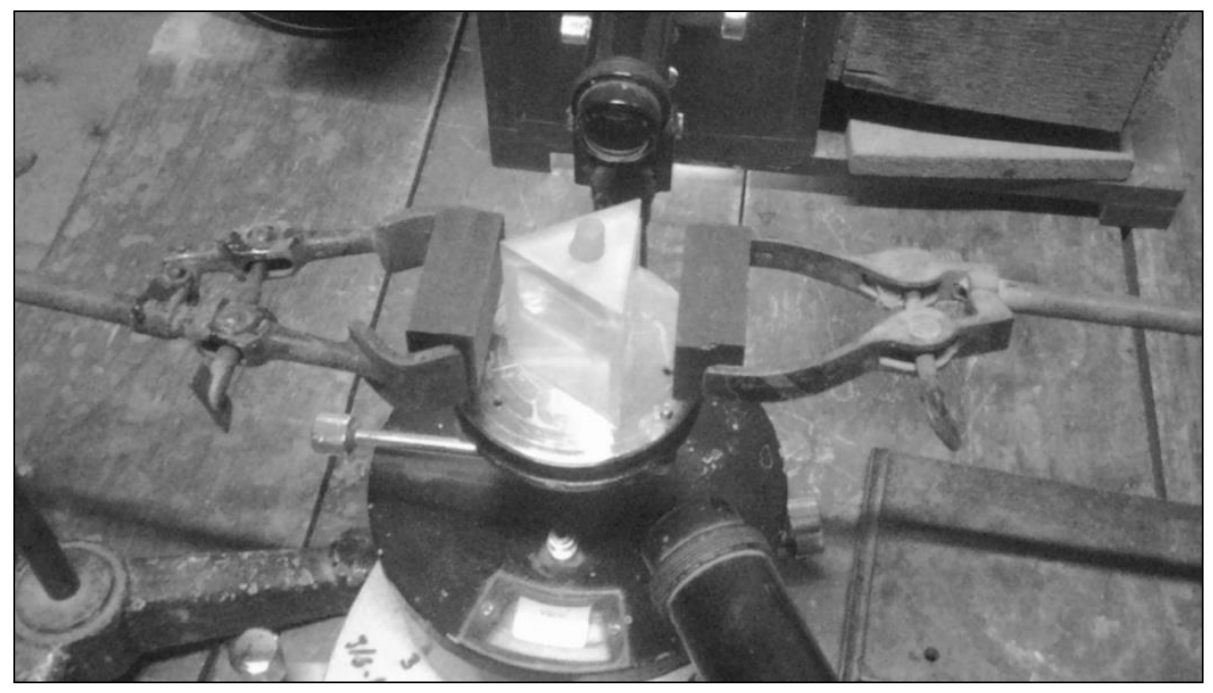

Figure 1: Experimental Setup to Obtain Refractive Index of Diamagnetic KCl Solution in Presence of non Uniform Magnetic Field

\begin{tabular}{|c|c|c|c|c|c|c|c|}
\hline Sr. & \multicolumn{2}{|c|}{ fm Position } & \multicolumn{2}{|c|}{ Direct Reading } & \multicolumn{2}{|c|}{ Difference } & Mean \\
\hline & $\mathrm{A}^{\prime}$ & $\mathrm{B}^{\prime}$ & $\mathrm{A}$ & B & $A^{\prime}-A$ & $\mathrm{~B}^{\prime}-\mathrm{B}$ & 〔m \\
\hline 1 & $154^{\circ} 47^{\prime}$ & $334^{\circ} 04^{\prime}$ & $130^{\circ} 45^{\prime}$ & $310^{\circ} 34^{\prime}$ & $24^{\circ} 02^{\prime}$ & $23^{\circ} 30^{\prime}$ & $23^{\circ} 46^{\prime}$ \\
\hline 2 & $142^{\circ} 56^{\prime}$ & $322^{\circ} 43^{\prime}$ & $118^{\circ} 18^{\prime}$ & $298^{\circ} 06^{\prime}$ & $24^{\circ} 38^{\prime}$ & $24^{\circ} 37^{\prime}$ & $24^{\circ} 37^{\prime} 30^{\prime \prime}$ \\
\hline 3 & $128^{\circ} 48^{\prime}$ & $308^{\circ} 39^{\prime}$ & $104^{\circ} 28^{\prime}$ & $284^{\circ} 16^{\prime}$ & $24^{\circ} 20^{\prime}$ & $24^{\circ} 23^{\prime}$ & $24^{\circ} 21^{\prime} 30^{\prime \prime}$ \\
\hline 4 & $117^{\circ} 06^{\prime}$ & $296^{\circ} 52^{\prime}$ & $92^{\circ} 41^{\prime}$ & $272^{\circ} 32^{\prime}$ & $24^{\circ} 25^{\prime}$ & $24^{\circ} 20^{\prime}$ & $24^{\circ} 22^{\prime} 30^{\prime \prime}$ \\
\hline 5 & $103^{\circ} 53^{\prime}$ & $283^{\circ} 42^{\prime}$ & $79^{\circ} 55^{\prime}$ & $254^{\circ} 45^{\prime}$ & $23^{\circ} 58^{\prime}$ & $23^{\circ} 57^{\prime}$ & $23^{\circ} 57^{\prime} 30^{\prime \prime}$ \\
\hline
\end{tabular}

\section{1] One Molal KCl Solution in Absence of Magnetic Field}


Hence refractive index of 1 molal $\mathrm{KCl}$ solution at room temperature is 1.3410

\section{2] Two Molal KCl Solution in Absence of Magnetic Field}

\begin{tabular}{|c|c|c|c|c|c|c|c|}
\hline Sr. & \multicolumn{2}{|c|}{ 'm Position } & \multicolumn{2}{c|}{ Direct Reading } & \multicolumn{2}{c|}{ Difference } & Mean \\
\hline & $\mathbf{A}^{\prime}$ & $\mathbf{B}^{\prime}$ & $\mathbf{A}$ & $\mathbf{B}$ & $\mathbf{A}^{\prime}-\mathbf{A}$ & $\mathbf{B} \mathbf{B}^{\prime}-\mathbf{B}$ & [m \\
\hline 1 & $94^{\circ} 57^{\prime}$ & $274^{\circ} 44^{\prime}$ & $69^{\circ} 55^{\prime}$ & $249^{\circ} 45^{\prime}$ & $25^{\circ} 02^{\prime}$ & $24^{\circ} 49^{\prime}$ & $24^{\circ} 55^{\prime}$ \\
\hline 2 & $81^{\circ} 30^{\prime}$ & $261^{\circ} 22^{\prime}$ & $56^{\circ} 40^{\prime}$ & $236^{\circ} 05^{\prime}$ & $24^{\circ} 50^{\prime}$ & $25^{\circ} 17^{\prime}$ & $25^{\circ} 03^{\prime}$ \\
\hline 3 & $66^{\circ} 50^{\prime}$ & $246^{\circ} 40^{\prime}$ & $41^{\circ} 47^{\prime}$ & $121^{\circ} 44^{\prime}$ & $25^{\circ} 03^{\prime}$ & $24^{\circ} 56^{\prime}$ & $24^{\circ} 59^{\prime}$ \\
\hline 4 & $51^{\circ} 08^{\prime}$ & $231^{\circ} 13^{\prime}$ & $26^{\circ} 35^{\prime}$ & $206^{\circ} 37^{\prime}$ & $24^{\circ} 33^{\prime}$ & $24^{\circ} 36^{\prime}$ & $24^{\circ} 34^{\prime} 30^{\prime}{ }^{\prime}$ \\
\hline 5 & $30^{\circ} 39^{\prime}$ & $318^{\circ} 43^{\prime}$ & $14^{\circ} 02^{\prime}$ & $194^{\circ} 12^{\prime}$ & $24^{\circ} 37^{\prime}$ & $24^{\circ} 31^{\prime}$ & $24^{\circ} 34^{\prime}$ \\
\hline
\end{tabular}

$$
\begin{aligned}
\int \mathrm{m} & =25^{\circ} 05^{\prime} \\
\mu & =2 * \sin \left\{\left[\mathrm{A}+\int \mathrm{m}\right] / 2\right\} \\
\mu & =1.3522
\end{aligned}
$$

Hence refractive index of 2 molal $\mathrm{KCl}$ solution at room temperature is 1.3522

\section{3 ] 3 molal KCl solution in absence of magnetic field}

\begin{tabular}{|c|c|c|c|c|c|c|c|}
\hline Sr. & \multicolumn{2}{|c|}{ [m Position } & \multicolumn{2}{c|}{ Direct Reading } & \multicolumn{2}{c|}{ Difference } & Mean \\
\hline & $\mathbf{A}^{\prime}$ & $\mathbf{B}^{\prime}$ & $\mathbf{A}$ & $\mathbf{B}$ & $\mathbf{A}^{\prime}-\mathbf{A}$ & $\mathbf{B}$ '-B & 'm \\
\hline 1 & $21^{\circ} 5^{\prime}$ & $192^{\circ} 25^{\prime}$ & $346^{\circ} 36^{\prime}$ & $166^{\circ} 47^{\prime}$ & $25^{\circ} 39^{\prime}$ & $25^{\circ} 38^{\prime}$ & $25^{\circ} 38^{\prime} 30^{\prime}{ }^{\prime}$ \\
\hline 2 & $0^{\circ}$ & $180^{\circ} 15^{\prime}$ & $234^{\circ} 24^{\prime}$ & $154^{\circ} 40^{\prime}$ & $25^{\circ} 36^{\prime}$ & $25^{\circ} 35^{\prime}$ & $25^{\circ} 35^{\prime} 30^{\prime}$ \\
\hline 3 & $347^{\circ} 51^{\prime}$ & $168^{\circ} 09^{\prime}$ & $322^{\circ} 11^{\prime}$ & $142^{\circ} 32^{\prime}$ & $25^{\circ} 40^{\prime}$ & $25^{\circ} 37^{\prime}$ & $25^{\circ} 38^{\prime} 30^{\prime}$ \\
\hline 4 & $334^{\circ} 09^{\prime}$ & $154^{\circ} 26^{\prime}$ & $308^{\circ} 33^{\prime}$ & $128^{\circ} 54^{\prime}$ & $25^{\circ} 36^{\prime}$ & $25^{\circ} 32^{\prime}$ & $25^{\circ} 34^{\prime}$ \\
\hline
\end{tabular}

$$
\begin{aligned}
\{\mathrm{m} & =25^{\circ} 36^{\circ} 30^{\prime \prime} \\
\mu & =2^{*} \sin \{[\mathrm{A}+[\mathrm{m}] / 2\} \\
\mu & =1.3589
\end{aligned}
$$

Hence refractive index of 3 molal $\mathrm{KCl}$ solution at room temperature is 1.3589

\section{4] Four Molal Kcl Solution In Absence Of Magnetic Field}

\begin{tabular}{|c|c|c|c|c|c|c|c|}
\hline Sr. & \multicolumn{2}{|c|}{ dm Position } & \multicolumn{2}{c|}{ Direct Reading } & \multicolumn{2}{c|}{ Difference } & Mean \\
\hline & $\mathbf{A}^{\prime}$ & $\mathbf{B}^{\prime}$ & A & B & A'-A $^{\prime}$ & B'-B & [m \\
\hline 1 & $125^{\circ} 28^{\prime}$ & $305^{\circ} 07^{\prime}$ & $99^{\circ} 26^{\prime}$ & $279^{\circ} 06^{\prime}$ & $26^{\circ} 02^{\prime}$ & $26^{\circ} 01^{\prime}$ & $26^{\circ} 01^{\prime} 30^{\prime}{ }^{\prime}$ \\
\hline 2 & $109^{\circ} 22^{\prime}$ & $289^{\circ} 10^{\prime}$ & $83^{\circ} 34^{\prime}$ & $263^{\circ} 12^{\prime}$ & $25^{\circ} 48^{\prime}$ & $25^{\circ} 58^{\prime}$ & $25^{\circ} 53^{\prime}$ \\
\hline 3 & $94^{\circ} 54^{\prime}$ & $274^{\circ} 36^{\prime}$ & $69^{\circ} 08^{\prime}$ & $248^{\circ} 58^{\prime}$ & $25^{\circ} 46^{\prime}$ & $25^{\circ} 38^{\prime}$ & $25^{\circ} 42^{\prime}$ \\
\hline 4 & $85^{\circ} 20^{\prime}$ & $265^{\circ} 15^{\prime}$ & $59^{\circ} 33^{\prime}$ & $239^{\circ} 24^{\prime}$ & $25^{\circ} 47^{\prime}$ & $25^{\circ} 51^{\prime}$ & $25^{\circ} 49^{\prime}$ \\
\hline 5 & $67^{\circ} 54^{\prime}$ & $247^{\circ} 49^{\prime}$ & $41^{\circ} 66^{\prime}$ & $221^{\circ} 18^{\prime}$ & $26^{\circ} 38^{\prime}$ & $26^{\circ} 31^{\prime}$ & $26^{\circ} 34^{\prime} 30^{\prime}$ \\
\hline
\end{tabular}

$$
\begin{aligned}
\int \mathrm{m}=26^{\circ} & \\
\mu & =2 * \sin \{[\mathrm{A}+\{\mathrm{m}] / 2\} \\
\mu & =1.3639
\end{aligned}
$$

Hence refractive index of 4 molal $\mathrm{KCl}$ solution at room temperature is 1.3639 
5] One Molal Kcl Solution in Presence of Non Uniform Magnetic Field

\begin{tabular}{|c|c|c|c|c|c|c|c|}
\hline $\mathrm{Sr}$ & \multicolumn{2}{|c|}{ dm Position } & \multicolumn{2}{|c|}{ Direct Reading } & \multicolumn{2}{|c|}{ Difference } & Mean \\
\hline & $\mathbf{A}^{\alpha}$ & B' & $\mathbf{A}$ & B & $A^{\prime}-A$ & B'-B & $\int \mathrm{m}$ \\
\hline 1 & $31^{\circ} 08^{\prime}$ & $211^{\circ} 12^{\prime}$ & $6^{\circ} 40^{\prime}$ & $186^{\circ} 46^{\prime}$ & $24^{\circ} 28^{\prime}$ & $24^{\circ} 26^{\prime}$ & $24^{\circ} 27^{\prime}$ \\
\hline 2 & $349^{\circ} 29^{\prime}$ & $169^{\circ} 47^{\prime}$ & $324^{\circ} 59^{\prime}$ & $145^{\circ} 14^{\prime}$ & $24^{\circ} 30^{\prime}$ & $24^{\circ} 33^{\prime}$ & $24^{\circ} 31^{\prime} 30^{\prime \prime}$ \\
\hline 3 & $338^{\circ} 19^{\prime}$ & $158^{\circ} 37^{\prime}$ & $313^{\circ} 48^{\prime}$ & $134^{\circ} 08^{\prime}$ & $24^{\circ} 31^{\prime}$ & $24^{\circ} 29^{\prime}$ & $24^{\circ} 30^{\prime}$ \\
\hline 4 & $320^{\circ} 41^{\prime}$ & $141^{\circ} 01^{\prime}$ & $296^{\circ} 30^{\prime}$ & $116^{\circ} 48^{\prime}$ & $24^{\circ} 11^{\prime}$ & $24^{\circ} 13^{\prime}$ & $24^{\circ} 12^{\prime} 30^{\prime \prime}$ \\
\hline 5 & $305^{\circ} 25^{\prime}$ & $125^{\circ} 43^{\prime}$ & $281^{\circ} 20^{\prime}$ & $101^{\circ} 38^{\prime}$ & $24^{\circ} 05^{\prime}$ & $24^{\circ} 05^{\prime}$ & $24^{\circ} 05^{\prime}$ \\
\hline
\end{tabular}

$$
\begin{aligned}
\{\mathrm{m} & =24^{\circ} 21^{\prime} \\
\mu & =2 * \sin \{[\mathrm{A}+[\mathrm{m}] / 2\} \\
\mu & =1.3427
\end{aligned}
$$

6] Two Molal KCl Solution in Presence of Non Uniform Magnetic Field

\begin{tabular}{|c|c|c|c|c|c|c|c|}
\hline Sr & \multicolumn{2}{|c|}{ 'm position } & \multicolumn{2}{c|}{ Direct reading } & \multicolumn{2}{c|}{ difference } & mean \\
\hline & $\mathbf{A}^{\prime}$ & $\mathbf{B}^{\prime}$ & $\mathbf{A}$ & $\mathbf{B}$ & $\mathbf{A}^{\prime}-\mathbf{A}$ & $\mathbf{B}^{\prime}-\mathbf{B}$ & dm \\
\hline 1 & $122^{\circ} 21^{\prime}$ & $302^{\circ} 04^{\prime}$ & $97^{\circ} 27^{\prime}$ & $277^{\circ} 10^{\prime}$ & $24^{\circ} 54^{\prime}$ & $24^{\circ} 54^{\prime}$ & $24^{\circ} 54^{\prime}$ \\
\hline 2 & $107^{\circ} 24^{\prime}$ & $287^{\circ} 34^{\prime}$ & $82^{\circ} 49^{\prime}$ & $262^{\circ} 35^{\prime}$ & $24^{\circ} 35^{\prime}$ & $24^{\circ} 59^{\prime}$ & $24^{\circ} 47^{\prime}$ \\
\hline 3 & $94^{\circ} 23^{\prime}$ & $274^{\circ} 06^{\prime}$ & $69^{\circ} 28^{\prime}$ & $249^{\circ} 17^{\prime}$ & $24^{\circ} 55^{\prime}$ & $24^{\circ} 49^{\prime}$ & $24^{\circ} 53^{\prime}$ \\
\hline 4 & $80^{\circ} 47^{\prime}$ & $260^{\circ} 41^{\prime}$ & $55^{\circ} 43^{\prime}$ & $235^{\circ} 38^{\prime}$ & $25^{\circ} 04^{\prime}$ & $25^{\circ} 03^{\prime}$ & $25^{\circ} 03^{\prime} 30^{\prime}$ \\
\hline 5 & $66^{\circ} 15^{\prime}$ & $246^{\circ} 09^{\prime}$ & $41^{\circ} 20^{\prime}$ & $221^{\circ} 17^{\prime}$ & $24^{\circ} 55^{\prime}$ & $24^{\circ} 52^{\prime}$ & $24^{\circ} 53^{\prime} 30^{\prime}{ }^{\prime} \mathrm{s}$ \\
\hline
\end{tabular}

$$
\begin{aligned}
\int \mathrm{m} & =24^{\circ} 54^{\prime} \\
\mu & =2 * \sin \{[\mathrm{A}+[\mathrm{m}] / 2\} \\
\mu & =1.3498
\end{aligned}
$$

7] Three Molal KCl Solution in Presence of non Uniform Magnetic Field

\begin{tabular}{|c|c|c|c|c|c|c|c|}
\hline Sr & \multicolumn{2}{|c|}{ dm Position } & \multicolumn{2}{c|}{ Direct Reading } & \multicolumn{2}{c|}{ Difference } & Mean \\
\hline & $\mathbf{A}^{\prime}$ & $\mathbf{B}^{\prime}$ & $\mathbf{A}$ & $\mathbf{B}$ & $\mathbf{A}^{\prime}-\mathbf{A}$ & $\mathbf{B} \mathbf{B}^{\prime}-\mathbf{B}$ & [m \\
\hline 1 & $276^{\circ} 17^{\prime}$ & $96^{\circ} 34^{\prime}$ & $250^{\circ} 49^{\prime}$ & $71^{\circ} 01^{\prime}$ & $25^{\circ} 28^{\prime}$ & $25^{\circ} 30^{\prime}$ & $25^{\circ} 29^{\prime}$ \\
\hline 2 & $259^{\circ} 38^{\prime}$ & $79^{\circ} 50^{\prime}$ & $234^{\circ} 10^{\prime}$ & $54^{\circ} 20^{\prime}$ & $25^{\circ} 28^{\prime}$ & $25^{\circ} 30^{\prime}$ & $25^{\circ} 29^{\prime}$ \\
\hline 3 & $228^{\circ} 08^{\prime}$ & $48^{\circ} 03^{\prime}$ & $202^{\circ} 37^{\prime}$ & $22^{\circ} 33^{\prime}$ & $25^{\circ} 31^{\prime}$ & $25^{\circ} 30^{\prime}$ & $25^{\circ} 30^{\prime} 30^{\prime}$ \\
\hline 4 & $32^{\circ} 25^{\prime}$ & $212^{\circ} 30^{\prime}$ & $6^{\circ} 30^{\prime}$ & $186^{\circ} 40^{\prime}$ & $25^{\circ} 55^{\prime}$ & $25^{\circ} 50^{\prime}$ & $25^{\circ} 52^{\prime} 30^{\prime}$ \\
\hline 5 & $300^{\circ} 40^{\prime}$ & $120^{\circ} 56^{\prime}$ & $275^{\circ} 27^{\prime}$ & $95^{\circ} 48^{\prime}$ & $25^{\circ} 13^{\prime}$ & $25^{\circ} 08^{\prime}$ & $25^{\circ} 10^{\prime} 30^{\prime}$ \\
\hline
\end{tabular}

$$
\begin{aligned}
\{\mathrm{m} & =25^{\circ} 30^{\prime} \\
\mu & =2^{*} \sin \{[\mathrm{A}+[\mathrm{m}] / 2\} \\
\mu & =1.3576
\end{aligned}
$$




\section{8] Four Molal KCl Solution in Presence of non Uniform Magnetic Field}

\begin{tabular}{|c|c|c|c|c|c|c|c|}
\hline \multirow[t]{2}{*}{$\mathrm{Sr}$} & \multicolumn{2}{|c|}{ dm Position } & \multicolumn{2}{|c|}{ Direct Reading } & \multicolumn{2}{|c|}{ Difference } & \multirow{2}{*}{$\begin{array}{c}\text { Mean } \\
\text { dm }\end{array}$} \\
\hline & $\mathbf{A}^{\prime}$ & B' & $\mathbf{A}$ & B & $\mathbf{A}^{\prime}-\mathbf{A}$ & B'-B & \\
\hline 1 & $200^{\circ} 15^{\prime}$ & $20^{\circ} 09^{\prime}$ & $174^{\circ} 30^{\prime}$ & $354^{\circ} 17^{\prime}$ & $25^{\circ} 45^{\prime}$ & $25^{\circ} 52^{\prime}$ & $25^{\circ} 48^{\prime} 30^{\prime \prime}$ \\
\hline 2 & $186^{\circ} 06^{\prime}$ & $5^{\circ} 56^{\prime}$ & $160^{\circ} 19^{\prime}$ & $340^{\circ} 06^{\prime}$ & $25^{\circ} 47^{\prime}$ & $25^{\circ} 50^{\prime}$ & $25^{\circ} 48^{\prime} 30^{\prime \prime}$ \\
\hline 3 & $172^{\circ} 12^{\prime}$ & $352^{\circ}$ & $326^{\circ} 11^{\prime}$ & $146^{\circ} 30^{\prime}$ & $25^{\circ} 42^{\prime}$ & $25^{\circ} 49^{\prime}$ & $25^{\circ} 45^{\prime} 30^{\prime \prime}$ \\
\hline 4 & $157^{\circ} 29$ & $337^{\circ} 16^{\prime}$ & $131^{\circ} 45^{\prime}$ & $311^{\circ} 30^{\prime}$ & $25^{\circ} 44^{\prime}$ & $25^{\circ} 46^{\prime}$ & $25^{\circ} 45^{\prime}$ \\
\hline 5 & $137^{\circ} 37^{\prime}$ & $137^{\circ} 37^{\prime}$ & $111^{\circ} 30^{\prime}$ & $111^{\circ} 13^{\prime}$ & $46^{\circ} 07^{\prime}$ & $26^{\circ} 07^{\prime}$ & $26^{\circ} 07^{\prime}$ \\
\hline
\end{tabular}

$\int \mathrm{m}=25^{\circ} 50^{\prime}$

$\mu=2 * \sin \left\{\left[\mathrm{A}+\int \mathrm{m}\right] / 2\right\}$

$\mu=1.3618$

\section{RESULT AND DISCUSSION}

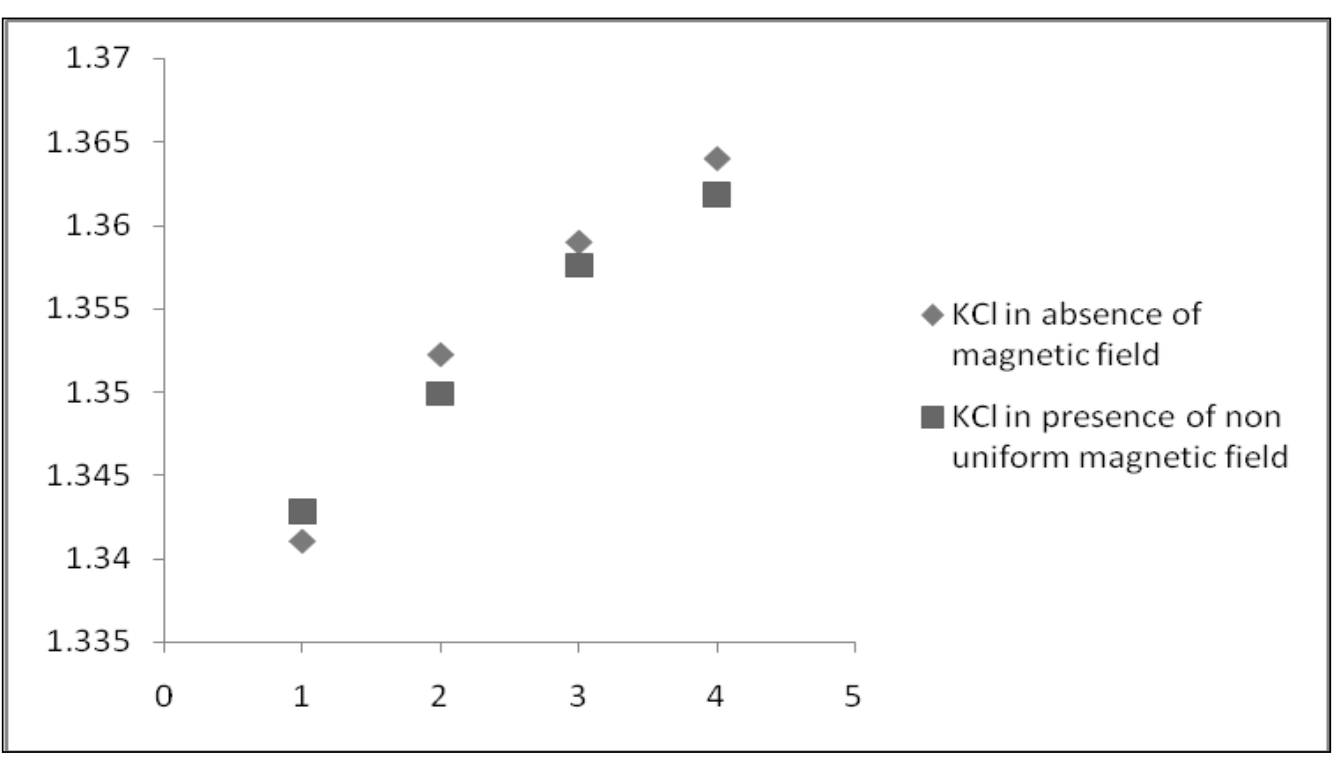

Figure 2: Graph of Refractive Index of Medium (y axis) Against Molal Concentration (x axis)

Figure (2) denotes graph of refractive index of $\mathrm{KCl}$ solution in absence and in presence of non uniform magnetic field against molal concentration. It was clearly seen that refractive index of one molal $\mathrm{KCl}$ solution is greater in presence of non uniform magnetic field than in absence of magnetic field. The increase in refractive index of $1 \mathrm{molal} \mathrm{KCl}$ solution in presence of non uniform magnetic field may be due to contamination of medium or solution might have larger temperature or maybe due to instrumental error [7] [8]. It was also observed that refractive index of diamagnetic $\mathrm{KCl}$ solution at 2, 3 and 4 molal concentration was smaller in presence of non uniform magnetic field than in absence of magnetic field. This was observed due to the tendency of diamagnetic matter to move from region of higher magnetic field to region of lower magnetic field [1] to form non uniform density in medium. In accordance with Fermat principle [3] and relation between refractive index and velocity of light in medium [6] it was concluded that refractive index of diamagnetic $\mathrm{KCl}$ solution decreases when placed in non uniform magnetic field. 


\section{CONCLUSIONS}

Refractive index of diamagnetic $\mathrm{KCl}$ solution reduces when placed in non uniform magnetic field due to change in magnetic moment of diamagnetic atom [2]. From graph, it was concluded that refractive index of diamagnetic $\mathrm{KCl}$ solution in presence and in absence of magnetic field increases with increase in molal concentration of $\mathrm{KCl}$ solution.

\section{Future Scope}

The conclusion can be verified for various diamagnetic solutions at different temperature and different applied magnetic field. Refractive index of medium can be studied for diamagnetic solution placed in uniform magnetic field too.

\section{REFERENCES}

1. Doubtnut. (Sept19, 2020). Magnetism and matter. Retrieved from (https://www.google.com/url?sa=t\&source=web\&rct=j\&url=https://www.doubtnut.com/question-answer-physics/assertionwhen-diamagnetic-material-is-placed-in-a-non-uniform-magnetic-held-it-tends-to-move-from-s30626171\&ved=2ahUKEwjdgOyMscrxAhVSgtgFHZn2ANgQFjAFegQIIBAF\&usg=AOvVaw14qoSfQz5QQJ7N5r3JcP78)

2. Griffith David Jeffrey. (1981) Magnetic field in matter. Introduction to electrodynamics: Pearson Cambridge University, 1981, Pages599, ISBN 978-1108420419

3. Oettler J 'et al ' (2013) Fermat's Principle of Least Time Predicts Refraction of Ant Trails at Substrate Borders. PLoS ONE 8(3): e59739. doi:10.1371/journal.pone.0059739

4. Yangang Liu \& Peter Daum, (2008). Brookhaven National Laboratory, Atmospheric Science division, USA.Retrieved from https://doi.org/10.1016/j.jaerosci.

5. Zhu Xingyu 'et al' (ICMMTA 2016). Relationship between refractive index and molar concentration of multi component solution.jupiter_zhu_007@126.com

6. Varsity Tutors. MCAT Physical: Velocity and index of refraction. Retrieved from (https://www.google.com/url?sa=t\&source=web\&rct=j\&url=https://www.varsitytutors.com/mcat_physical-help/velocity-andindex-ofrefraction\&ved=2ahUKEwidtpWpt8rxAhWrH7cAHcY5ALQQFjAWegQIHhAC\&usg=AOvVaw3Z8bnwTlaPi0232oTqI4rN\&cs hid $=1625436788348)$

7. University of Toronto. Refractive index Theory. Retrieved from (https://www.google.com/url?sa=t\&source=web\&rct=j\&url=https://www.utsc. utoronto.ca/webapps/chemistryonline/producti on/refractive.php\%23: :text\%3DRefractive\%2520index\%2520values\%2520are\%2520usually,due\%2520to\%2520a\%2520sma ller\%2520ratio.\&ved=2ahUKEwjVs5uCuMrxAhUIxTgGHaRQCH4QFjANegQIAxAF\&usg=AOvVaw2KkYKXGujH2OvBMyiZhby\&cshid=1625436986606)

8. Harald Wack. (April 1 2009). Limitations of refractive index: contamination can artificially decrease readings. Retrieved from (https://www.google.com/url?sa=t\&source=web\&rct=j\&url=https://www.thefreelibrary.com/Limitations\%2Bof\%2Brefractiv e\%2Bindex\%253A\%2Bcontamination\%2Bcan\%2Bartificially...a0198185272\&ved=2ahUKEwiSq_mHucrxAhV1xzgGHc9zCOsQFjAAegQIAxAC\&usg=AOvVaw1S8v4oY3WIqQ1DBpd75IOd \&cshid=1625437437101) 
9. McGraw-Hill Dictionary of Scientific \& Technical Terms, 6E. (2003). Retrieved from https://encyclopedia2.thefreedictionary.com/Schuster+method

10. Maharashtra State Board Class 11 physics textbook. Retrieved (2019)

11. Sharma, Sanjeev, et al. "Temperature Dependence ZnS Based One-Dimensional Photonic Crystals." International Journal of Physics And Research (IJPR) ISSN (P) : 2250-0030.

12. Abdullah, H. I., K. J. Kadhim, and I. H. Hilal. "Optical properties of methyl methacrylate photo polymerization initiated by nanosilver." Journal of Industrial Engineering and Research 5.2.

13. Saboo, Shweta, and Chandra Prakash Gupta. "Design Of Photonic Crystal Fiber For Minimum Confinement Loss By Varying The Size Of Holes." International Journal of Electronics and Communication Engineering (IJECE) 2.2.

14. Chakrabarty, Bishwajit S. "Evaluation of optical constants of wide band gap cadmium doped polypyrrole." International Journal of Research in Engineering \& Technology 2 : 37-44. 
